\title{
A Wide-angle Secondary Ion Probe for Organic Ion Imaging
}

\author{
Casey C. Grimm, R. T. Short, and Peter J. Todd \\ Oak Ridge National Laboratory, Oak Ridge, Tennessee, USA
}

\begin{abstract}
A secondary ion source has been developed for an organic ion microprobe capable of imaging samples up to $2 \mathrm{~cm}$ in diameter. The source uses a focused $5 \mathrm{keV} \mathrm{Cs}{ }^{+}$ion beam which is rastered across the sample surface, and secondary ions from each point on the sample are collected and formed into a low energy beam to be analyzed by a quadrupole mass filter. Dynamic emittance matching is employed to deflect ions from off-axis points on the sample back onto the mass analyzer axis. Rastering and dynamic emittance matching are rapidly controlled by assembly language programs using an IBM/AT (80286) type computer. A low energy ion monitor was used to tune and evaluate the secondary ion source by providing a magnified cross-sectional image of the ion beam at the source exit aperture. A well-focused and centered secondary ion beam was obtained from each point on the sample, indicating that large-scale dynamic emittance matching with high collection efficiency is possible. Mass resolved images of grids and glycerol samples are shown to demonstrate the performance of the integrated secondary ion source mass analyzer and control system. (J Am Soc Mass Spectrom 1991, 2, 362-371)
\end{abstract}

S econdary ion mass spectra (SIMS spectra) of organic compounds often contain pseudomolecular ions characteristic of the organic sample, particularly when SIMS spectra are obtained under conditions of low primary ion dose $\left(<10^{14}\right.$ primary ions $/ \mathrm{cm}^{2}$ ), so-called static SIMS conditions $[1,2]$. This observation leads to the notion that an organic ion microprobe could be developed that would be capable of determining the spatial distribution of organic compounds in various matrices, particularly biological tissue. By using microprobes primarily designed for inorganic samples, and under conditions of high primary dose, or "dynamic SLMS" conditions, others have reported obtaining spatial distributions of organic compounds in various matrices [3], chromatographic media [4], and deposited neat on metal grids [5] and under grids [6]. As discussed below, these results suggest that performance criteria for a microprobe for biomedical applications are different from those for microprobes designed for inorganic analysis, and organic analysis in other matrices. Because very little is known about sample preparation, ion yields from biological tissue, etc., our philosophy in designing a microprobe for biomedical applications has been to strive for high sensitivity through high secondary ion transmission and relaxed spatial resolution requirements.

Spatial resolution of secondary ion microprobes is

Address reprint requests to Peter I. Todd, Oak Ridge National Laboratory, Oak Ridge, TN 37831-6365. wholly determined by the parameters of the primary ion beam: the size of the primary spot focused onto the sample and the ability to move precisely that spot to various positions on the sample. By using liquid metal ion guns, which are capable of producing primary ion spots as small as $20 \mathrm{~nm}$, distributions of ${ }^{14} \mathrm{CN}$ in labeled chromosomes have been determined with submicron spatial resolution [7]. A consequence of restricting the primary dose to below $10^{14}$ ions $/ \mathrm{cm}^{2}$, however, is low count rate and poor contrast. For the general problem of determining spatial distributions of organic compounds in biological tissue, it is unreasonable to believe that useful spatial resolution of 20 $\mathrm{nm}$ can be obtained. There are three reasons for this pessimism. First, organic compounds, which comprise the bulk of dried protoplasm, suffer significant damage from the primary ion beam used to create the secondary ions used for analysis [6]. With neat samples under dynamic SIMS conditions, there is an exponential decrease of characteristic ion intensity with time and primary dose, and a corresponding increase in intensity of ions that are not analytespecific [5]. To avoid obtaining data from regions that have been altered by damage, only a small fraction of each resolution element can actually be sampled. Second, toxins, drugs, and their metabolites are often biologically active at concentrations below $100 \mathrm{ppm}$. At $100 \mathrm{ppm}$, the probability that a targeted analyte molecule can be ionized, mass resolved, and detected from a 1- $\mu \mathrm{m}$ square resolution element becomes vanishingly low: only 100 target molecules would exist in 
the resolution element. Third, there is, as yet, no established method of sample preparation that is known to enhance secondary ion emission from a matrix like biological tissue. At least until such methods are developed, it is reasonable to design the microprobe such that even samples that do not yield a significant secondary ion current can be analyzed. Once even a weak signal is obtained, it can be used to test and optimize any method subsequently developed to improve secondary ion yield. In short, while ion optics can be designed for maximum transmission, which is one of the usual goals in mass spectrometer design, establishing a large resolution element is a design specification of the work here.

The problem with employing a large spatial resolution element is that the sample must be correspondingly large. For example, to generate an image consisting of $100 \times 100$ picture elements or pixels, each representing an area on the sample of $100-\mu \mathrm{m}$ square, the sample would need to be at least $1 \mathrm{~cm}^{2}$. For high transmission and good mass resolution, aperture requirements for quadrupole mass filters are roughly $\pm 100 \mu \mathrm{m}$ and $1^{\circ}$ divergence [8]. Thus, the freld of view of the microprobe may be approximately two orders of magnitude larger than the acceptance aperture of a quadruple mass filter. This means that only secondary ions from a small region of the sample can be transmitted through a quadrupole mass filter. There is no point in sacrificing spatial resolution for sensitivity if that gain in sensitivity is lost as a result of decreased transmission through the quadrupole mass filter.

A solution to problems of this type [9] was first demonstrated by Liebl [10] and termed dynamic emittance matching (DEM). The technique is based on the observation that with microprobes, at any given time, secondary ions are emitted from only a small area of the target, namely the position of the primary ion spot. Thus, it is only from that exact spot that ions need to be transmitted into the mass analyzer. By linking deflector potentials in the secondary ion source to the rastering deflector potentials of the primary ion gun, fields of view as large as $0.5 \mathrm{~mm}$ have been demonstrated.

For very large samples, the DEM approach must be extended. The capability for using nonlinear relationships between primary and secondary ion deflection, and the capability for secondary source refocusing are necessary for larger fields of view, such as $2-\mathrm{cm}$ in diameter. For such large primary ion displacement corrections must be performed by computer and done rapidly; if only $1 \mathrm{sec}$ were required to retune the instrument for each point on a $100 \times 100$ array, almost $3 \mathrm{hr}$ would be required to acquire an image. In short, the instrument must be self-tuning.

In this article we describe the design and testing of an organic ion microprobe designed to determine the spatial distributions of organic compounds in biological tissue samples. Because the microprobe source is the single most important component of the instrument, its performance is first evaluated by its ion optical behavior, and then as part of the integrated instrument under computer control. We have developed a special low energy monitor [11] that can be used to evaluate the ion optical behavior, and can use the well-known behavior of glycerol [12] and its solutions to show the capabilities of the integrated system.

\section{System Components}

Figure 1 is a schematic diagram of the instrument. The major components of the system include the cesium primary ion gun, the sample introduction system and target, the secondary ion source, quadrupole mass filter, detector, and computer system. Orientation of the primary ion gun is $45^{\circ}$ to the target normal, and the mean ion axis of secondary ion source and quadrupole mass filters is normal to the target surface. Primary ion raster is controlled by the computer via two 12-bit digital-to-analog (D/A) converters. Similarly, control of deflector plates of the secondary ion source for DEM is by a second pair of 12-bit D/A converters. Mass selection of the quadrupole mass filters proceeds from the computer via 16-bit $D / A$ converter to the radiofrequency oscillator/DC power supply to the $3 / 8$-in. quadrupole mass filter rods. Analog detection of secondary ion current is by a high voltage conversion dynode-continuous dynode electron multiplier-preamplifier system whose output is fed to a 12-bit analog-to-digital (A/D) converter and thence to the computer.

The vacuum system consists of a 6 -in. radius stainless steel sphere with various ports that employ high vacuum flanges. The sample handler, which is commercially available (GATAN, Inc., Pleasanton, CA), allows introduction of up to eight samples simultane-

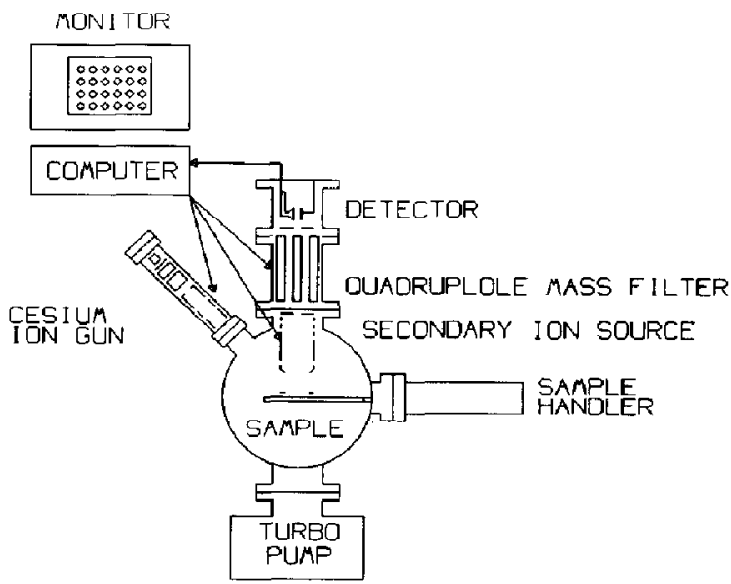

Figure 1. Schematic diagram of organic secondary ion microprobe. 
ously, and permits translational adjustment in three axes and rotational adjustment about the main axis of the handler. Pumping of the entire system is by a Balzers Model 510 Turbomolecular Pump (Balzers Aktiengesellschaft, Lichtenstein). With organic samples, operating pressure is ca. $10^{-6}$ torr; pressures below $10^{-7}$ torr have been achieved with inorganic samples. The quadrupole mass filter and detector assembly are mounted in their own vacuum housings, and can be attached in modular fashion, via common 6-in. diameter vacuum flanges.

Primary ion source. The primary ion source is comprised of a condenser or ion gun and an objective. The ion gun, obtained commercially (Phrasor Scientific, Duarte, CA) is a tetrode source, consisting of a thermionic $\mathrm{Cs}^{+}$source, extraction, focus, and ground electrode system. The principal features of this gun include its ability to generate a $3-\mathrm{mm}$ diameter monoenergetic beam of up to $22 \mathrm{keV} \mathrm{Cs}{ }^{+}$ions with a current density of $1-10 \mu \mathrm{A} / \mathrm{cm}^{2}$ at any distance from the source. For the experiments described here, $\mathrm{Cs}^{+}$ kinetic energy was limited to $5 \mathrm{keV}$ so as to be compatible with the electrostatic focusing capabilities of the objective lens.

The objective consists of a three-element einzel lens and raster plates. At a working distance of 2.5 $\mathrm{cm}$, primary ion spot size of $10 \mu \mathrm{m}$ can reportedly be achieved. By using a stainless steel grid of known wire diameter and spacing as a target, and comparing the known ratio of these dimensions to the ratio observed in the secondary ion image, the spot size of the beam could be determined. Under the conditions employed here, $45^{\circ}$ primary ion incidence and $6-\mathrm{cm}$ working distance, a spot size of about $100 \mu \mathrm{m}$ was indicated. A large working distance is necessary for a large raster of the primary ion beam across the sample, but this capability comes at the expense of spatial resolution.

For the experiment reported here, unless otherwise specified, a primary ion current of $100 \mathrm{pA}$ and current density of $1 \mu \mathrm{A} / \mathrm{cm}^{2}$ was employed. These parameters were measured with a shielded Faraday cup and picoammeter that could be inserted in the position normally occupied by the sample.

Secondary ion source. The secondary extraction and deceleration lens system was designed to have extremely high collection efficiency for organic secondary ions, which typically are emitted in a cosine distribution with energies of a few electronvolts or less. The secondary ion beam formed from ions sputtered at each point on the sample, exits the source along the quadrupole mean ion axis with minimum angular divergence so as to enhance transmission through the quadrupole mass filter. Kinetic energy of the exiting beam can be varied between 5 and $50 \mathrm{eV}$. The sample was held at ground potential for the experiments reported here, but a potential up to $200 \mathrm{~V}$ can be applied to it.

Major ion optical features of the cylindrically symmetric source are shown in Figure 2, which was prepared by using the optical program SIMION [13]. The

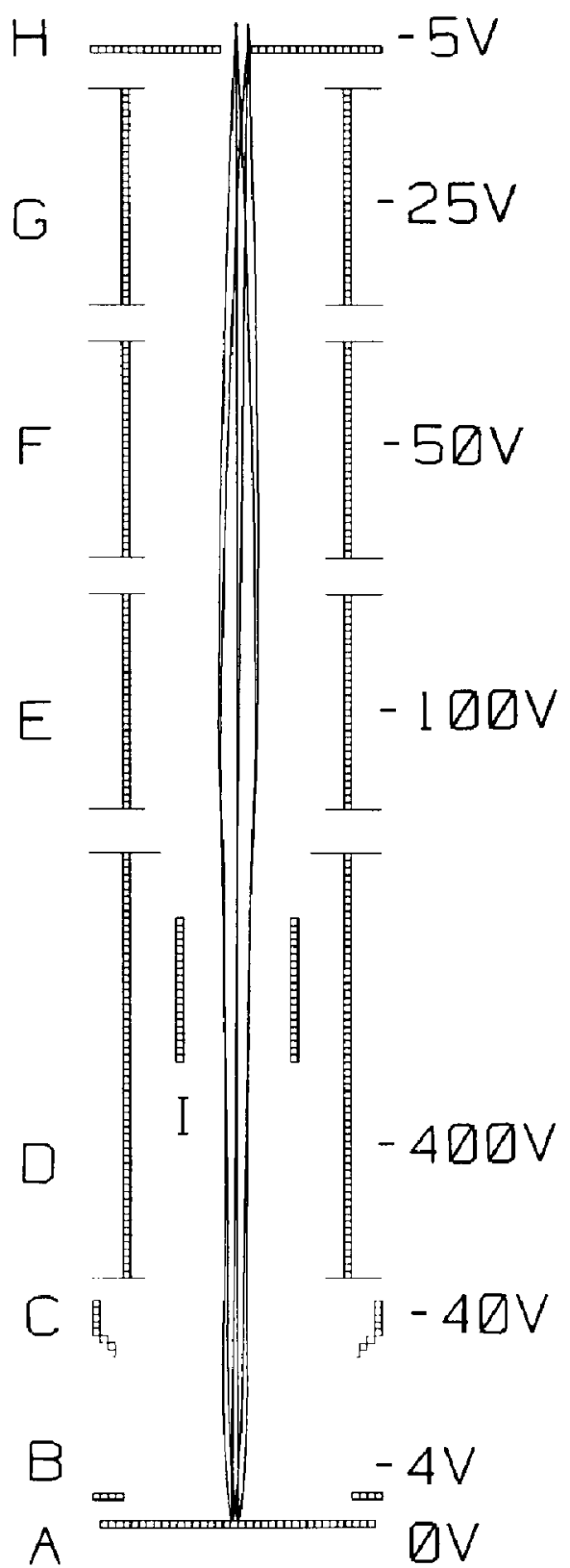

Figure 2. Schematic of secondary ion source with ray tracing calculations from SIMION. Elements are indicated as: $\mathrm{A}=$ target $/$ sample; $B=$ Wehnelt; $C=$ extraction focus; $D=$ extraction; $E-G=$ deceleration lens; $H=$ exit aperture; $I=$ deflectors. Calculations indicate a magnification of -2.5 for small displacements from the lens axis. 
source is a result of integration of a number of discrete ion optical elements. The Wehnelt [14] and extraction cone serve to modify the field at the periphery of the target so that secondary ions are forced toward the mean ion axis. The high voltage (400 V) acceleration element is efficient in keeping secondary ions near the mean ion axis, but the ions must be decelerated by the exponential field of the three-element deceleration lens to $5 \mathrm{eV}$ or so for entrance to the quadrupole mass filter. The location of the deflectors is such that all ions, regardless of origin, tend to cross the mean ion axis at the location of the deflectors; the mean voltage applied to the deflectors is that of the acceleration element $(-400 \mathrm{~V})$. For DEM, the fact that ions are at the mean ion axis at the point of deflection means that only the direction of the beam needs to be corrected, not the beam's displacement.

Trajectories shown in Figure 2 represent $1-\mathrm{eV}$ ions emitted from point sources at the center, and $0.1 \mathrm{~cm}$ from the center of the sample; initial velocity vectors are $0^{\circ}$ and and $\pm 20^{\circ}$ from the surface normal at each point; final beam energy is nominally $6 \mathrm{eV}$. These parameters are within the normal operating range of the instrument and the expected kinetic energy distribution of organic secondary ions. Magnification of the secondary ion source can be measured directly from Figure 2 as the ratio of radial displacement of trajectories at the exit of the source to the radial displacement of trajectories at the sample. The displacement at the exit of the source, or the image, corresponds to .25 $\mathrm{cm}$; the specified input parameters included an initial $0.1-\mathrm{cm}$ displacement at the target. This leads to a magnification of -2.5 . A $100-\mu \mathrm{m}$ diameter primary ion beam would result in a secondary beam diameter of $250 \mu \mathrm{m}$ at the source exit, neglecting aberrations. The negative sign arises because the image at the exit of the source is inverted with respect to the mean ion axis; this behavior is required for simple electrostatic lenses.

These calculations indicate that a well-behaved low energy beam suitable for entrance into a quadrupole mass analyzer can be formed from ions sputtered from a small spot at the center of the target. We recently showed that a well-focused beam of low energy ions projected onto the entrance of a quadrupole mass filter is a requirement for producing a well behaved beam, i.e., of small diameter and divergence, at the exit of a quadrupole mass filter [11]. Because the mass spectrometer under development will eventually include a collision cell and second quadrupole mass filter, it is necessary that the mass-resolved ions exiting the first quadrupole be well behaved.

The practical ion optical characteristics of the secondary ion source were tested using a special low energy ion monitor [11], which was attached at the exit of the source in place of the quadrupole shown in Figure 1. The monitor projects the cross-sectional image of the secondary ion beam at the source exit aperture onto a CRT, with approximately a sixfold magnification. With this device beam shape, size, and position (relative to the exit aperture) can be investigated as lens and target parameters are changed and as the primary ion beam was deflected to various points on the sample.

The secondary ion source was evaluated as follows. A tightly focused $5-\mathrm{keV} \mathrm{Cs}^{+}$primary beam was directed onto a flat graphite sample at the center of the secondary lens system. Graphite was chosen as a test sample because carbon clusters are desorbed with energies characteristic of organic secondary ions, but the graphite sample is less prone to damage than organic samples. The primary beam spot size was previously determined to be less than $200 \mu \mathrm{m}$ in diameter by using an $\mathrm{NaI}$ [15] sample and viewing the fluorescence spot with a telescope. The secondary source lens voltages were varied about the values predicted by SIMION calculations to focus the beam image which was projected onto the monitor CRT. The difference between a defocused secondary ion beam and a well-focused secondary ion beam, when both are viewed on the CRT of the low energy ion monitor, are shown in Figure $3 a$ and $3 b$, respectively. In Figure 3a, the beam illuminated the entire $0.64-\mathrm{cm}$ exit aperture, whereas a beam image corresponding to a secondary beam diameter of roughly $0.1 \mathrm{~cm}$ (Figure $3 b)$ was obtained for optimal focusing voltages. The shadow of a grid in the photographs arises from a grid at the monitor entrance apertures that was used to test the monitor. It was found that the lens parameters necessary to obtain a focused secondary beam spot (Figure $3 \mathrm{~b}$ ) agreed to within $10 \%$, in all cases, with calculations.

To obtain a measure of the secondary ion collection efficiency, the graphite sample was replaced with a glycerol sample. The amplified secondary ion current from the dual microchannel plate (MCP) assembly in the low energy ion monitor was measured as a function of the $5-\mathrm{keV} \mathrm{Cs}{ }^{+}$primary ion beam intensity. Secondary ion yield of glycerul for such a primary beam has been previously measured to be about $2 \%$ of the primary ion beam current [16]. The amplified secondary current was in this case measured to be 25 nA for a primary current of 3 pA. For $2 \%$ secondary ion emission, this measurement indicates a transmission efficiency of $50 \%(+50 \%,-25 \%)$ for the secondary ion source, if an MCP gain of $10^{6}$ is assumed. The uncertainty is primarily a consequence of uncertainty in the MCP gain.

The calculated behavior of secondary ions emitted at a distance of $1.0 \mathrm{~cm}$ from the axis of symmetry of the lens system is shown in Figure $4 a$. Initial ion energy for this calculation was assumed to be $0.5 \mathrm{eV}$; this is a different initial kinetic energy from $1 \mathrm{eV}$ chosen for Figure 1 and shows that the ion optics of the source work equally well for slightly different initial kinetic energies. Also in Figure 4, a greater number of trajectories are displayed, namely with 


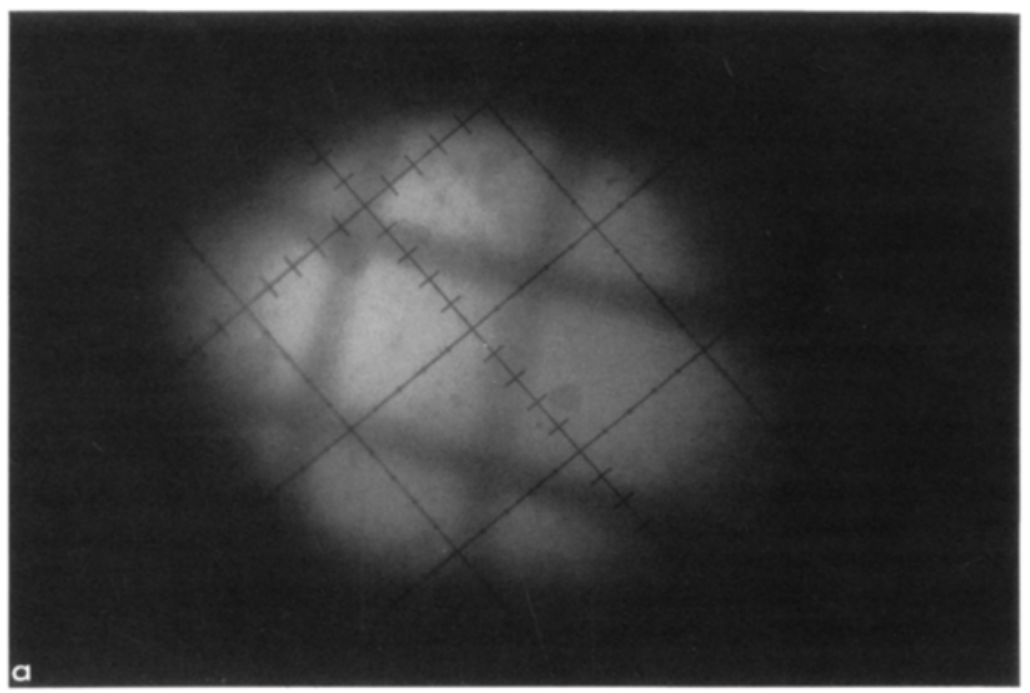

Figure 3. Magnified $(\mathrm{ca}, \times 6)$ image of the low energy ion beam exiting the (a) defocused and (b) focused secondary ion source as viewed on the CRT of the low energy beam monitor. The grid shadow is a result of a grid at the entrance of the monitor, placed there to enable monitor tuning.

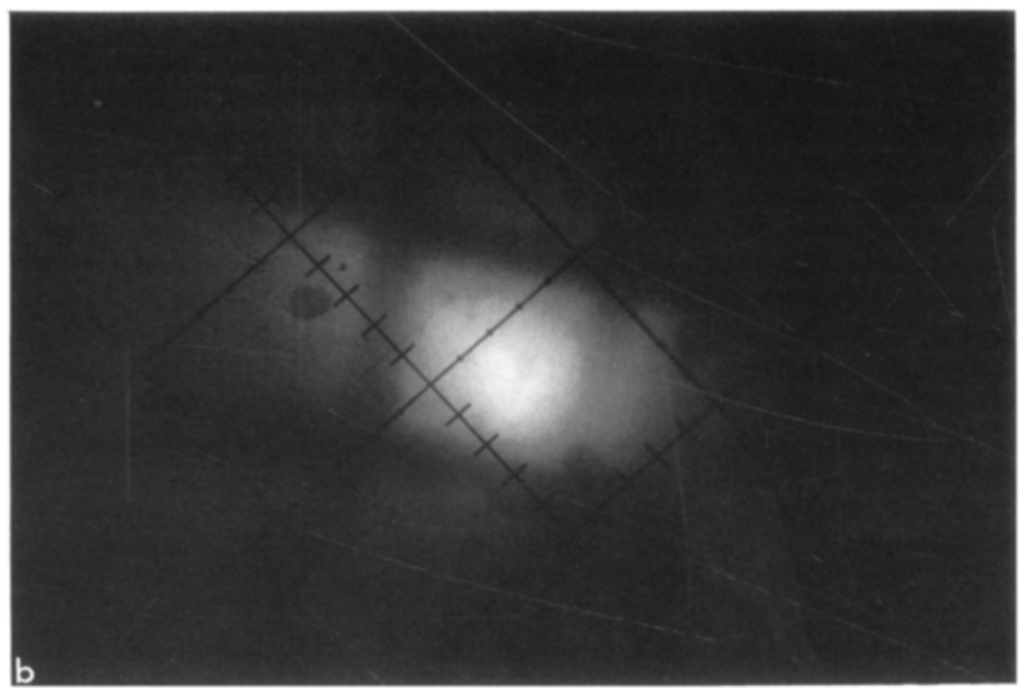

initial trajectories of $0^{\circ}, \pm 22.5^{\circ}$, and $\pm 45^{\circ}$ from the surface nurmal also assumed.

The need to deflect the ions back onto the lens axis (DEM) to have the ions exit the source in the center of the aperture is readily apparent from these trajectory calculations. For this purpose, deflection plates were placed in the field-free region near the middle of the extraction lens element where the ions have the highest kinetic energy. The position, shape, and potential of the two electrodes nearest the target were chosen such that a nearly parallel beam enters the deflection plate region. This improves the effectiveness of dynamic emittance matching because a nearly parallel on-axis beam then exits the deflection region. Large scale DEM should be possible, as indicated by Figure $4 \mathrm{~b}$, even for the extreme case where the secondary ions originate from the edge of a $2-\mathrm{cm}$ diameter sample.

As indicated earlier, a lens system with cylindrical symmetry, i.e., the lens elements are all symmetric about the mean ion axis, except for the deflectors, which of course, have planar symmetry, is represented in Figures 2 and 4. Programs such as SIMION do not permit the overlay of a planar symmetric lens onto a system of cylindrically symmetric lenses, except in the case of magnetic fields. To calculate the deflector voltages used to produce Figure 4, certain assumptions were made, based upon the well-known behavior of magnetic and electrostatic deflectors [17]. First is that in a field-free region, neither electrostatic nor magnetic deflectors have any first-order focusing effect if they are symmetric. Second, electrostatic and 


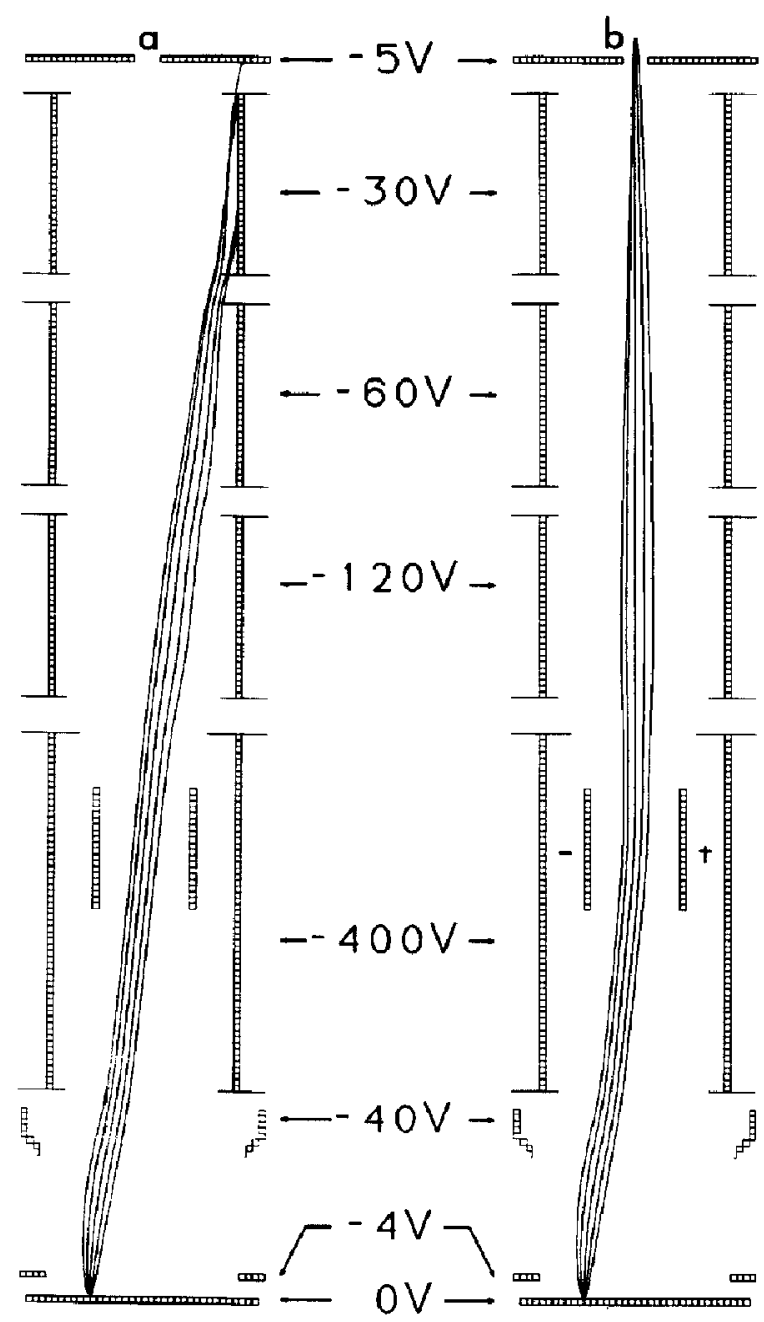

Figure 4. SMMION trajectory plot of secondary ions emitted from a point $1.0 \mathrm{~cm}$ from target center (a) without DEM and (b) with DEM employed.

magnetic deflection can be calculated from 1 and 2 .

$$
\tan \phi_{E}=(\delta V L) /\left(V_{0} d\right)
$$

where: $\phi_{E}-$ deflection angle

$\delta V=$ voltage difference between plates

$V_{0}=$ ion accelerating voltage

$L=$ length of the deflectors

$d=$ plage separation

and

$$
\tan \phi_{\mathrm{B}}=\mathbf{B} L /\left(2 V_{0} m\right)^{1 / 2}
$$

where: $\mathbf{B}=$ magnetic field strength

$m=$ mass of ion
By superimposing a magnetic field in the position occupied by the deflectors, an operation allowed by SIMION, and by observing the trajectory correction for a specified magnetic freld and ion mass, the appropriate deflection voltage for electrostatic deflection can be readily calculated. Despite the clumsiness of this approach, it is mathematically and physically correct; furthermore, observed deflection correction voltages are within $5 \%$ of calculated values. For example, in the specific case of secondary ion emission $1 \mathrm{~cm}$ from the target center, as shown in Figure $4 \mathrm{~b}$, the potential difference between deflector plates to correct the trajectory of the beam was calculated to be $60 \mathrm{~V}$, and the observed deflection voltage at optimal transmission was $60 \mathrm{~V}$.

The exiting beam in Figure $4 \mathrm{~b}$ appears very similar to that in Figure 2, where the ions originate from the target center. Potential contours are more curved near the edges of the sample, and therefore ions are more strongly focused in the acceleration lens. For this reason, in addition to trajectory correction by the deflectors, the deceleration lens must be made weaker. Ions emitted from the target extremity are not overfocused at the source exit in Figure $4 \mathrm{~b}$ because these corrections have been applied to the focusing voltages, i.e., the focusing voltages in Figure $4 b$ are different from those of Figure 2.

The behavior of the secondary ion source for ions originating far from the target center, as observed by using the low energy ion monitor, is shown in Figure 5. The CRT display, shown in Figure $5 a$, is observed without DEM employcd. The image obtained when the secondary ion source deflectors are used to correct for secondary ion emission displaced from the target center is shown in Figure 5b. The ability of the ion source to redirect the secondary ion beam onto the lens axis while maintaining a well-focused beam of similar intensity is shown in Figure 5. It was found that DEM was effective up to the edge of the sample holder $(0.6 \mathrm{~cm}$ off axis), indicating images should be possible for samples up to $1.2 \mathrm{~cm}$ in diameter. The size of the sample inlet system currently prohibits testing of samples larger than $1.2-\mathrm{cm}$ in diameter; however, with an improved sample inlet system it should be possible to image samples up to $2-\mathrm{cm}$ in diameter.

Slight coma aberration [17] was observed for secondary beams originating from the sample extremes, because the field curvature becomes increasingly less symmetric about the point of origin of secondary ions near the sample surface in this region. A clear need to adjust the deceleration lens parameters as the primary beam was deflected farther off axis was not found during this test. The effect may have been too small to observe from the cross-sectional images on the CRT. Aperture requirements of quadrupole mass analyzers may be more sensitive to overfocus in principle, but when the quadrupole mass filter was installed no 


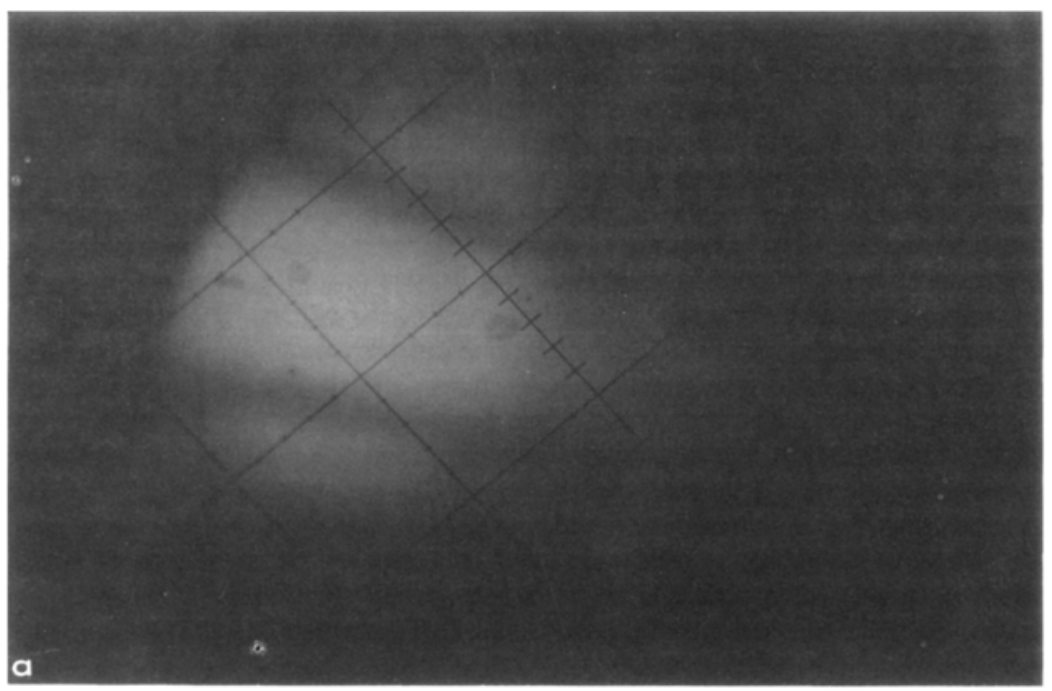

Figure 5. Magnified image of secondary ions emitted from source under conditions similar to those used for Figure 4a and $b$, respectively, as seen on low energy ion monitor CRT.

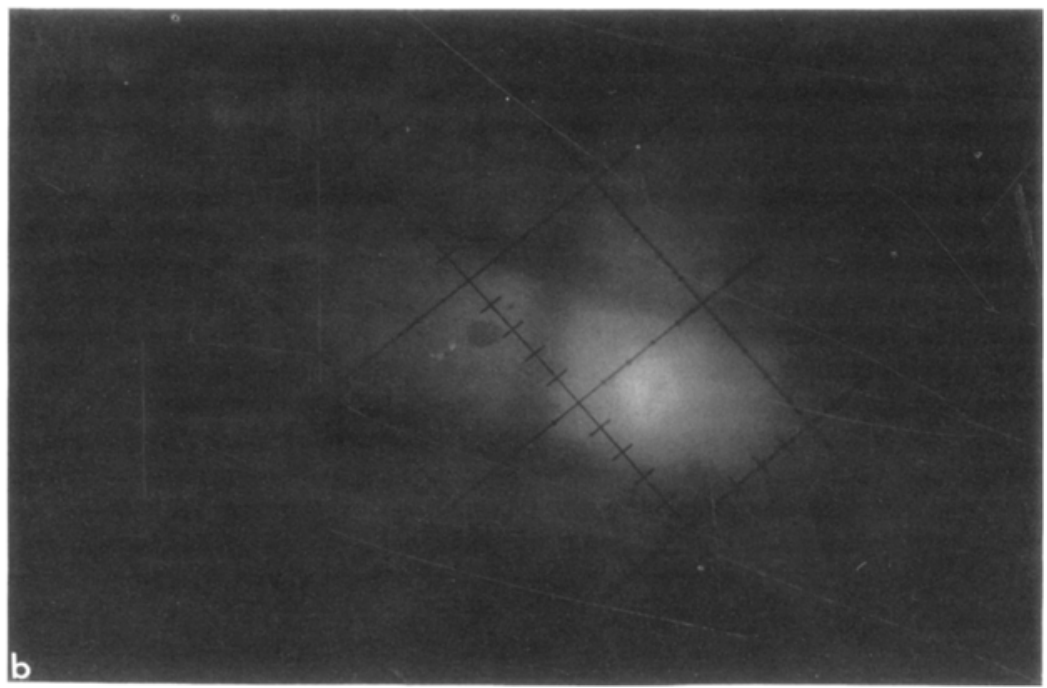

degradation of secondary ion intensity was evident for targets as large as $1-\mathrm{cm}$ in diameter.

\section{System Integration and Evaluation}

Computer control system. The data system is designed to maximize the rate of data acquisition and display update. For an image of $200 \times 200$ pixels, or 40,000 resolution elements, if the data acquisition time were only $100 \mathrm{~ms}$ per pixel, over an hour would be required to obtain an image. An IBM/AT (80286) based system, with a $16 \mathrm{MHz}$ clock and $10 \mathrm{MHz}$ microprocessor, was chosen as the central control and data acquisition unit. All programs were written in Assembly language in order not to software-limit the speed of instrument control. The interface (Burr-Brown Corporation, Tucson, AZ) consists of up to eight 12-bit $D / A$ converters, two $16-b i t D / A$ converters, and one
A/D converter with an eightfold differential miltiplex capability. The 12-bit D/As are configured such that values are luaded into ail registers sequentially, after which all $D / A s$ are triggered simultaneously. Settling time for the 12-bit D/As is $500 \mu \mathrm{s}$; for the 16-bit D/As, the settling time is $8 \mu \mathrm{s}$.

The rate at which a secondary ion image can be obtained is limited by the time required to change parameters such as deflector and lens potentials and the time necessary to detect a significant number of mass resolved secondary ions. For optimal speed, images based upon the intensities of a number of secondary ions are obtained by monitoring the intensity of ions of one mass for each image. The settling time of quadrupole mass filter power supplies is of the order of a few milliseconds, whereas the settling time of deflector and lens power supplies is a maximum of $500 \mu \mathrm{s}$. Because each sputtering event under 
static SDMS conditions is stochastic, a mass spectrum obtained at any point on the sample is independent of the sequence in which it was obtained.

The secondary ion beam current can be monitored in an analog mode, or pulse counting can be used for low intensity. In the analog mode, a 12-bit A/D samples the ion signal from an electron multiplier type detector and rapidly sums repetitive samples to improve signal-to-noise levels. Up to 1000 readings can be acquired and summed in less than $20 \mathrm{~ms}$. Consequently, image acquisition rate is limited by the settling time of the $D / A s$ and the secondary ion intensity, not by computer speed nor quadrupole power supply settling time.

For each point on the sample (Primary $X$ and $Y$ deflector plate voltages) there will be unique settings for the secondary $X$ and $Y$ deflection plate voltages for optimum transmission of secondary ions. Optimal secondary ion source voltages may also vary with position of the primary ion spot on the sample, as SIMION calculations indicate. The set of optimal values for primary and secondary potentials are determined from an automated tuning routine. Seventeen primary $\mathrm{X}$ and $\mathrm{Y}$ deflector settings and the corresponding "starting search value" for the secondary $X$ and $Y$ deflectors settings are stored in a table. In general, these values come from previous data acquisitions, although the initial set of values was determincd manually using the low energy beam monitor [11]. The primary ion beam is positioned on the target by using an arbitrary set of deflector settings. The secondary deflector voltages are varied around the "starting search value" to optimize the secondary ion signal. Upon location of the maximum signal, the values are stured and the primary beam is directed to the next point on the target and the procedure is then repeated for each of the remaining sets of deflector settings. A linear least squares fit of optimal deflector settings for which recorded intensity is above a specified threshold is then used to determine a slope and intercept for both the $\mathrm{X}$ and $\mathrm{Y}$ deflectors, along with a correlation value for each fit. This procedure can be repeated with the new values being used as the new "starting search values" each time, thus allowing an iterative procedure to be employed until correlation values reach an acceptable level. By using the linear relationships, secondary $X$ and $Y$ deflector settings can be calculated for any given point on the target.

A flat target is required for the calibration and the signal must be sufficiently noise free that small increments in the voltages applied to the deflector plates are discernible. To improve the signal-to-noise ratio, signal averaging is employed with from 1000 to 10,000 samples averaged per setting. Increasing the number of samples averaged from 1000 to 10,000 increases the time of the procedure from $30 \mathrm{sec}$ to $15 \mathrm{~min}$. For samples that are not flat, optimal primary and secondary potentials can, in principle, be determined directly from the sample. A linear relationship be- tween primary and secondary deflector settings would not be expected, except in the differential limit. In such cases, care must be taken that the static SIMS dose limit is not exceeded during tuning.

\section{Evaluation of Integrated System}

Tests of the secondary ion source optics and control system consisted of installing a quadrupole mass analyzer in place of the low energy ion monitor, and obtaining secondary ion images from samples of known shape. A hexagonal grid, placed over a $0.9-\mathrm{cm}$ in diameter hole in the sample positioning probe, was chosen as the first test sample because an image of a grid is a good measure of image resolution and distortion. A continuous dynode electron multiplier was placed at the exit of the quadrupole to monitor transmitted secondary ion current. With the mass analyzer in the radiofrequency-only mode, secondary ions above mass 20 , emitted from the sample grid, were detected. The image shown in Figure 6 was obtained in approximately $80 \mathrm{sec}$ and consists of $200 \times 200$ pixels. The amplified secondary ion current was sampled 64 times at each pixel for signal averaging. Relative intensities at each pixel are displayed as different shades of gray from white (low intensity) to black (high intensity). The actual grid dimensions are $75-\mu \mathrm{m}$ wire width, and $1900-\mu \mathrm{m}$ diagonal across each hexagon. From these ratios the primary ion spot was determined to be, at worst, $100-\mu \mathrm{m}$ in diameter. The

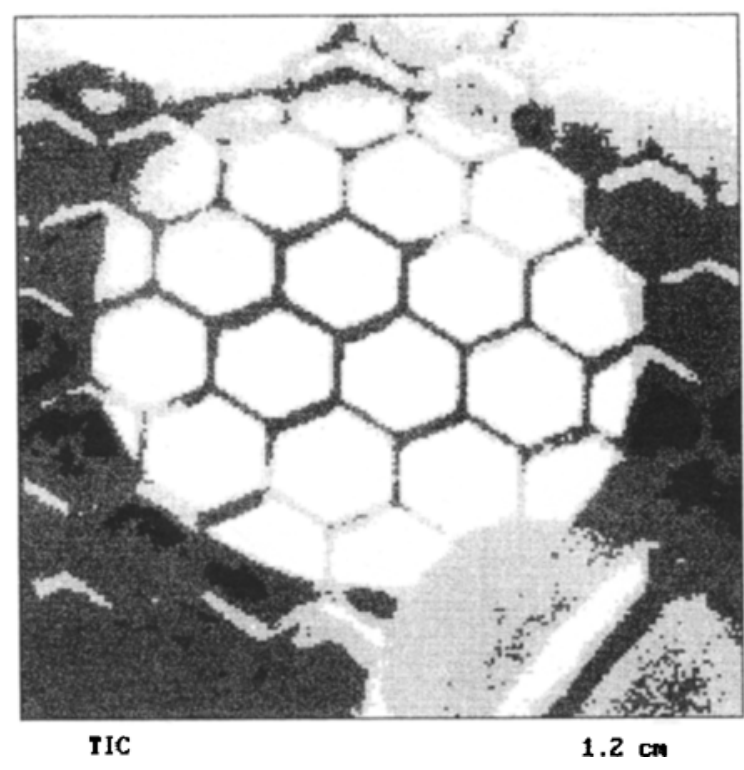

Figure 6. Secondary ion image of a hexagonal stainless steel grid, based upon total ions transmitted through the quadrupole in radiofrequency-only mode. Wire diameter of the grid is 75 $\mu \mathrm{m}_{i}$ spacing between wires is $1900 \mu \mathrm{m}$. Field of view is 1.2 $\mathrm{cm} / 1.2 \mathrm{~cm}$. Note the image of the screw head in lower right corner. 


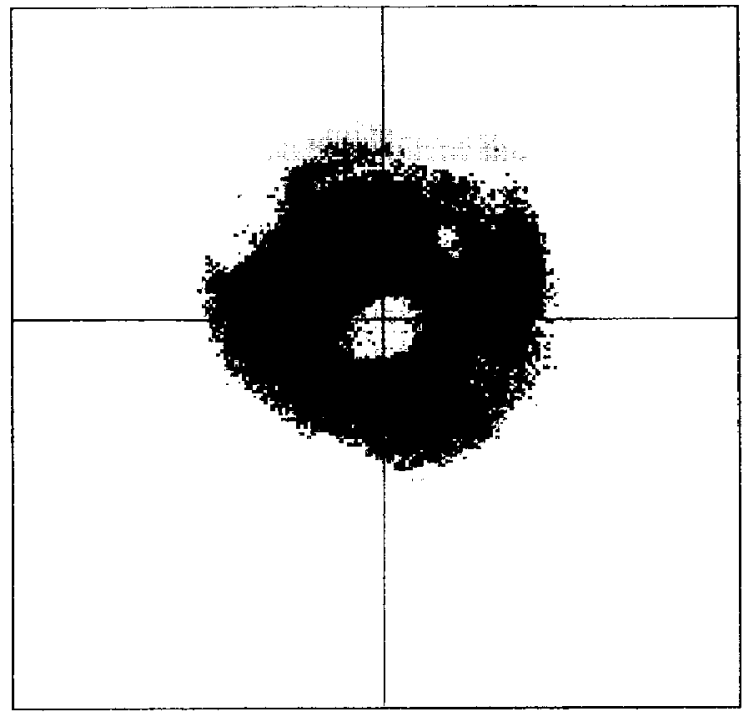

$m / z 185$ $1.2 \mathrm{~cm}$

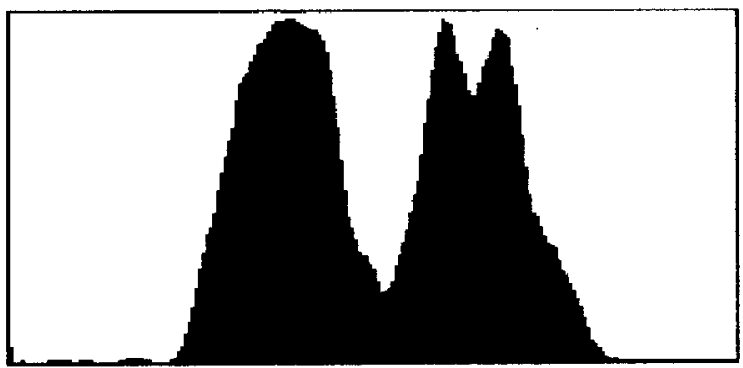

Figure 7. Secondary ion image of $m / z 185$ (protonated glycerol dimer) from a droplet of pure glycerol onto which was placed a crystal of arginine hydrochloride. The plot in the lower portion reflects the intensity of the targeted ion across the horizontal cursor indicated in the micrograph.

field of view is roughly $1.2 \mathrm{~cm} \times 1.2 \mathrm{~cm}$. The nearly uniform distribution of secondary ion emission across the image attests to the nearly uniform transmission of secondary ions regardless of origin.

The grid shown in Figure 6 is necessary to demonstrate that the requisite secondary ion image can be obtained. However, focusing and DEM for atomic secondary ions require less precision than for organic ions because the initial kinetic energy distribution of atomic ions is much larger than of organic ions. Ions with large energy spreads cannot be focused as sharply as ions with small energy spreads. Thus, DEM for atomic ions need not be as fine-tuned as when the technique is applied to organic ions. Secondary images taken from glycerol solutions are shown in Figures 7 and 8.

The ability of glycerol to permit long-lasting secondary ion emission is well known [12]. The sec-

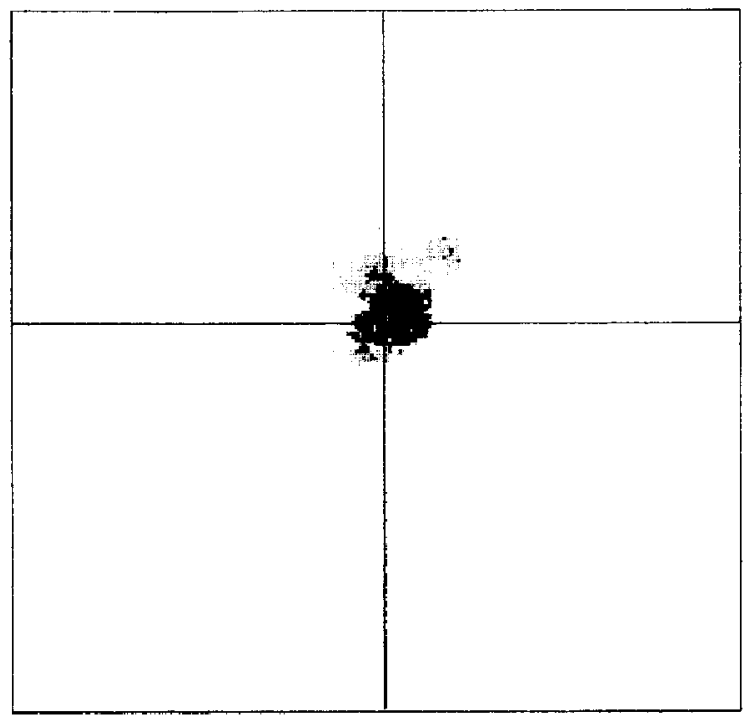

$m / 2175$

$1.2 \mathrm{~cm}$

Figure 8. Secondary ion image of $m / z 175$, protonated arginine, from the same sample used for Figure 7 . Note the complementary relationship between protonated arginine and the proton bound dimer of glycerol.

ondary ion images of $\mathrm{m} / \mathrm{z} 185$ (protonated glycerol dimer) and $m / z 175$ (protonated arginine) from a sample of glycerol onto which was placed a crystal of arginine hydrochloride are shown in Figures 7 and 8 . respectively. Below the micrographs in each figure are graphic plots of the intensity of the targeted ion, along the horizontal indicated by the crosshairs in the micrograph. About $80 \mathrm{sec}$ were required to acquire the data for each of the micrographs shown in Figures 7 and 8.

Figures 7 and 8 are unremarkable except for two features. First, the location of the arginine crystal in the sample was not visible to the naked eye. Second, the graphs in the lower portions of each of the figures are complementary and provide a quantitative measure of distribution that may not be readily apparent from the micrographs themselves. While this sort of information is of only passing interest for the exemplary samples, with biological samples, the co-location of targeted compounds is of significant value. 


\section{Summary}

The success of organic ion imaging of biological tissue pivots heavily on having a very high collection and transmission efficiency of secondary ions, as well as having rapid and precise control of the microprobe parameters and data acquisition. The secondary ion source presented in this article has been shown to have very high secondary ion collection efficiency and effective dynamic emittance matching over large areas. This latter feature is indicated by relatively uniform transmission through a quadrupole mass analyzer over the entire sample area. Mass resolved images up to $1.2-\mathrm{cm}$ in diameter (and most probably up to $2-\mathrm{cm}$ in diameter) can be rapidly obtained by using an efficient computer control system based on an IBM/AT (80286) computer. To obtain images of targeted organic compounds in biological tissue (in which ions at every mass are typically present), however, tandem mass spectrometry will have to be employed. A collision region and second quadrupole mass analyzer are currently under design and fabrication. The demonstrated focusing capabilities of the secondary ion source described here, and the reported behavior of ion transmission through quadrupoles $[8,11]$, bode well for its success.

With a spatial resolution of about $100 \mu \mathrm{m}$, the device described here does not exactly fit the criterion of $10 \mu \mathrm{m}$ spatial resolution that is technically required for a device to be a microprobe [9]. In this regard two points should be made. First is that spatial resolution can be improved over what we have reported here provided sufficient sensitivity and specificity in secondary ion analysis can be achieved. Second, as demonstrated in Figures 7 and 8 , organic secondary ion images can provide useful information, even from samples that are an order of magnitude larger than those that can be accommodated by "real" microprobes.

\section{Acknowledgments}

This research was sponsored by the U.S. Department of Energy, Office of Basic Energy Sciences, under Contract DE-AC05$840 R 21400$ with Martin Marietta Energy Systems, and by the National Institutes of Health under grant GM-41617. CCG gratefully acknowledges receipt of a postdoctoral fellowship sponsored by Oak Ridge Associated Universities.

\section{References}

1. Benninghoven, A.; Jaspers, D.; Sichtermann, W. Appl. Phys, 1976, 11, 35 ,

2. Grade, H.; Winograd, N.; Cooks, R. G.; J. Amer. Chem. Soc. 1977, 99, 7725 .

3. Schuetzle, D.; Prater, T. J,; deVries, J. E.; Bayly, A.; Vohrlik, P. Rev. Sct. Inst. 1988, 60, 53

4. DiDonato, G. G.; Busch, K. L. Anal. Chem. 1986, $58,3231$.

5. Gillen, G. L.; Simons, D. S.; Williams, P. Anal. Chem. 1990, 62,2122

6. Cole, R. B.; Guenat, C.; Hass, J. R.; Linton, R. W. Anal. Chem. 1987, 59, 1930.

7. Hallegot, P.; Girod, C.; LeBeau, M. M.; Levi-Setti, R. In Secondary Ion Mass Spectrometry-SIMS VI; Benninghoven, A; Evans, C. A.; McKeegan, K. D.; Storms, H. A.; Werner, H. W., Eds. Wiley: Chichester, 1990.

8. Dawson, P. H. In Quadrupole Mass Spectrometry; Dawson, P. H., Ed.; Elsevier: Amsterdam, 1976; p. 23.

9. Benninghoven, A.; Rudenauer, F. G., Werner, H. W. Secondary Ion Mass Spectrometry; Wiley: New York, 1987; pp. 442-462, 556.

10. Liebl, H. U.S. Patent 3,517,191.

11. Short, R. T.; Grim, C. C.; Todd, P. J. J. Amer. Soc. Mass Spectrom. 1991, 2, 226.

12. Barber, M.; Bordoli, R.; Elliott, R. D.; Sedgwick, R. D.; Tyler, A. N. Arzal. Chem. 1982, 54, 645A.

13. Dahl, D. A.; Delmore, I. E. The SIMION PC/PS2 User's Manual, Version 4; Informal Report EGG-CS-7233, Rev. 2, Idaho Falls National Engineering Laboratory, 1988.

14. Klemperer. O.; Barnett, M. E. Electron Optics; Cambridge, 1971; pp 320-325.

15. Todd, P. J. Org. Mass Spectrom 1988, 23, 419.

16. Todd, P. J. J. Amer. Soc. Mass Spectrom 1991, 2, 33.

17. Grivet, P. Electron Optics, Second English Edition; Hawkes, P. W., Trans.; Pergamon: Oxford, 1972, 159. 